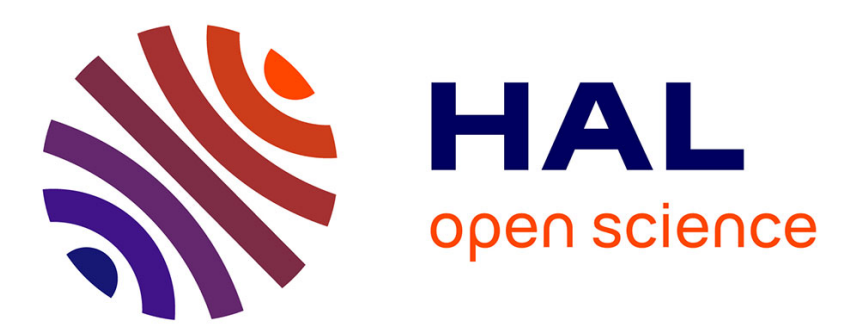

\title{
A New Method for Inter-Cell Interference Estimation in Uplink SC-FDMA Networks
}

\author{
Fatima Zohra Kaddour, Emmanuelle Vivier, Mylène Pischella, Philippe
}

Martins

\section{> To cite this version:}

Fatima Zohra Kaddour, Emmanuelle Vivier, Mylène Pischella, Philippe Martins. A New Method for Inter-Cell Interference Estimation in Uplink SC-FDMA Networks. VTC Spring 2012, May 2012, Yokohama, Japan. pp.1-5. hal-00822482

\section{HAL Id: hal-00822482 \\ https://hal.science/hal-00822482}

Submitted on 14 Oct 2014

HAL is a multi-disciplinary open access archive for the deposit and dissemination of scientific research documents, whether they are published or not. The documents may come from teaching and research institutions in France or abroad, or from public or private research centers.
L'archive ouverte pluridisciplinaire HAL, est destinée au dépôt et à la diffusion de documents scientifiques de niveau recherche, publiés ou non, émanant des établissements d'enseignement et de recherche français ou étrangers, des laboratoires publics ou privés. 


\title{
A New Method for Inter-Cell Interference Estimation in uplink SC-FDMA Networks
}

\author{
Fatima Zohra Kaddour ${ }^{1,2}$, Emmanuelle Vivier ${ }^{2}$, Mylene Pischella ${ }^{3}$, Philippe Martins ${ }^{1}$ \\ ${ }^{1}$ Telecom Paris Tech, 46 rue Barrault, Paris, France \\ 2 ISEP, 28 rue N-D des Champs, Paris, France \\ ${ }^{3}$ CNAM, 292 rue Saint-Martin, Paris, France
}

\begin{abstract}
This paper addresses a power control and InterCell Interference (ICI) estimation problem in uplink Single Carrier Frequency Division Multiple Access (SC-FDMA) networks. We consider a power control based on Signal to Noise and Interference Ratio (SINR) target to be achieved, which requires knowledge of the interference level. We propose a simple method to estimate ICI in uplink SC-FDMA networks, less greedy than the classical Monte Carlo method which has higher computation complexity. Numerical results validate the new method in multiple environments even after adding fast fading and shadowing effect and show its robustness against variation of these parameters.
\end{abstract}

Keywords: Uplink power control, Inter-Cell Interference (ICI) estimation, Single Carrier Frequency Division Multiple Access (SC-FDMA) networks.

\section{INTRODUCTION}

The 3GPP Long Term Evolution (LTE) represents a major advance in cellular technology. LTE offers significant improvements over previous technologies by introducing a new physical layer and reforming the core network. The main reasons for these changes in the Radio Access Network (RAN) system design are the need to improve the average user throughput (in order to reach peak data rates of $100 \mathrm{Mbits} / \mathrm{s}$ in downlink and $50 \mathrm{Mbits} / \mathrm{s}$ in the uplink) and the coverage area [1]. To achieve these targets, Orthogonal Frequency Division Multiple Access (ODFMA) was adopted for the LTE downlink and Single Carrier Frequency Division Multiple Access (SCFDMA) for the LTE uplink. These methods were selected in order to provide higher spectral efficiency, lower delay, high immunity to inter-symbol interference, good bandwidth scalability, and more multi-user flexibility than the currently deployed networks [2].

The radio air interface must be able to provide both high peak bit rates and acceptable cell-edge bit rates, even to cell edge users who are the most interfered by Inter-Cell Interference (ICI), i.e by users using the same radio resource in neighboring cells [3]. This problem is specially noticeable on the uplink, where ICI generated by mobile users must be evaluated.

The existing techniques to mitigate the level of inter-cell interference rely on two possible radio resource allocation techniques: (i) subcarrier allocation, which consists in determining a proper frequency planning (ii) power control: the transmission power must be as low as possible to achieve the target throughput while generating the least possible interference. Power control in SC-FDMA has been studied in the literature. An open-loop fractional power control has been considered in [4] and inter-cell power control in [5]. In [6] the authors propose to decrease or increase the transmit power by $1 \mathrm{~dB}$ if needed. A radio resource management using the cooperation of base stations is detailed in [7]. The power allocation presented in our study is based on the Signal to Interference plus Noise Ratio target $S I N R_{\text {target }}$ required by users and their environment (path loss, noise, ICI...). To perform this power control, we need to know the inter-cell interference generated at the base station level. The ICI determination can be done by estimating the transmission channel, as it is done in [8], where pilots are distributed in the transmitted signal. This idea is shared by the authors of [9] in the Rayleigh-type variable channels. [10] and [11] propose an analytical method based on collision probability distribution to analyze the ICI.

In this paper, we present a new method to estimate the interference caused by neighboring cells in the uplink case. We consider that the interference received by the base station of the central cell in the total allocated bandwidth is equal to the received power from a virtual point situated at the barycenter of each interfering sector, radiating with a median power. The way to compute this median power is exhibited. This method is simple to be implemented on simulator and is less greedy than classical Monte Carlo simulations which require higher computation complexity. The remainder of the paper is organized as follows. Section II introduces the system model and notations. Section III presents the new method for estimating inter-cell interference. Performance results are represented in Section IV. Conclusions are given in the last section.

\section{SySTEM MODEL}

We consider an LTE network composed of 19 cells: one central cell and tow rings of interference cells (i.e 18 cells). We focus on the uplink transmission, where a SC-FDMA technique is used. To simplify the problem, we assume that each user is assigned one Resource Block (RB), which is described by $N_{s c}^{R B}$ adjacent subcarriers and $N_{s y m b}^{U L}$ SC-FDMA symbols where $N_{s c}^{R B}=12$ and $N_{s y m b}^{U L}=6$ (case of normal cyclic prefix). The adjacency property allows us to average the Signal to Interference plus Noise Ration $S I N R$ over the whole used band, since the fast fading on adjacent subcarriers is correlated, instead of computing it by subcarrier as in 
OFDMA. Therefore, the $S I N R_{\text {target }}$ can be defined for each RB. For the sake of fairness, its value will be the same for all users. To mitigate interference, an appropriate frequency planning is required. Using a tri-sectored antenna located at the center of the hexagonal cell, we use three frequency bandwidths $B$, one for each sector of each cell (as illustrated on Figure 1).

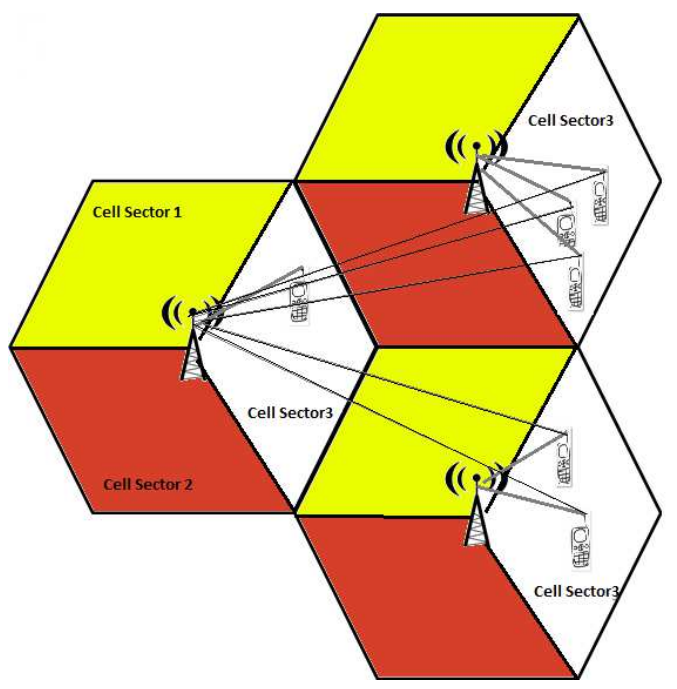

Fig. 1. Frequency planning with tri-sectored antennas - Example of interference in the uplink

The total interference $I_{c}$ suffered by the central Base Station (BS) is the sum of the interferences suffered by this BS from each neighboring cells, i.e:

$$
I_{c}=\sum_{k=2}^{19} I_{k c}
$$

where $I_{k c}$ is the interference caused by the neighboring sector $k$ to the central cell $c$. The computation of $I_{k c}$ will be detailed in section III.

The model uses power control to determine the transmission power of each user, so as to reach the $S I N R_{\text {target }}$. In addition, we set a throughput threshold that users can not overstep, even if their radio conditions could enable them to achieve more. Thus, every mobile station (MS) $j$ of the central cell is going to transmit on the resource block $l$, at the power $P_{e m_{j}}^{l}$ obtained after power control as follows:

$$
P_{e m_{j}}^{l}=\min \left[\frac{\operatorname{SINR}_{\text {target }} *\left(N+I_{c}\right)}{G_{t} * G_{r}\left(\theta_{j c}\right) * P_{L}\left(r_{j c}\right)}, P_{\text {max }}\right]
$$

where,

- $N$ represents the noise in the used frequency band. In practice, the noise is predominantly thermal.

- $G_{t}=1$ is the mobile's transmission antenna gain.

- $G_{r}$ is the BS antenna gain (used for reception) which depends on $\theta_{j c}$ : the angle between the mobile $j$ situated in the central cell and the main lobe of the central cell's BS radiation antenna pattern [13]:

$$
G_{r}\left(\theta_{j c}\right)=-\min \left[12 *\left(\frac{\theta_{j c}}{\theta_{3 d B}}\right)^{2}, A_{m}\right]
$$

where $\theta_{j c} \in\left[-60^{\circ}, 60^{\circ}\right], \theta_{3} d B$ is the $3 \mathrm{~dB}$ beamwidth which is $70^{\circ}$ for tri-sectored cell and $A_{m}=20 \mathrm{~dB}$ is the maximum attenuation [14].

- $P_{L}$ is the path loss depending of $r_{j c}$, the distance between the mobile $j$ and the base station of the central cell $c$.

$$
P_{L}\left(r_{j c}\right)=10^{-\frac{a}{10}} * r_{j c}^{-\frac{b}{10}}
$$

By using the Okumura Hata model [15], we obtain the coefficients $a=136.7$ and $b=34.4$.

- $P_{\max }$ is the maximum mobile transmission power, set to $125 \mathrm{~mW}$, i.e. $21 \mathrm{dBm}[19]$. .

\section{NEW METHOD OF ICI ESTIMATION}

Our objective is to develop an easier method than Monte Carlo simulations to estimate the LTE uplink inter-cell interference.

As it is illustrated on Figure 2, we consider that the contribution of each sector is equivalent to the power received at the central base station from a point situated at the barycenter of the interfering sector and radiating at $P_{m}$, where $P_{m}$ is the median power of $P_{e m_{j}}^{l}, j=1, \ldots, M_{S}, M_{S}$ representing the number of active users in the sector.

It follows that,

$$
I_{k c}=P_{m} * G_{t} * G_{r}\left(\theta_{k c}\right) * P_{L}\left(r_{k c}\right),
$$

where,

- $\theta_{k c}$ is the angle between the barycenter of the interfering sector and the main lobe of the central cell's BS antenna pattern $\left(\theta_{k c} \in\left[-180^{\circ}, 180^{\circ}\right]\right)$.

- $r_{k c}$ is the distance between the barycenter of the interfering sector and the central cell's BS.

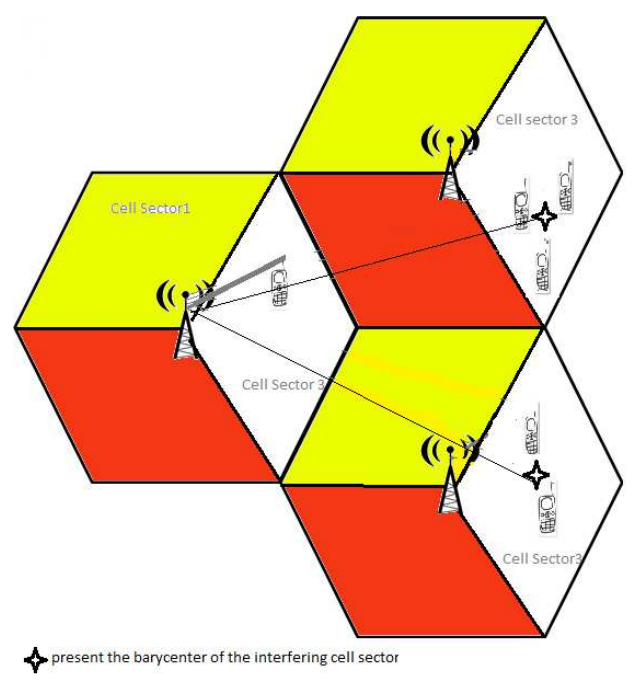

Fig. 2. ICI estimation using a virtual point situated at the barycenter of interfering sectors 
To be more fair, we allocate to users the same number of RB per time slot and we suppose that they try to reach the same $S I N R_{\text {target }}$. In order to mitigate interference, we reduce the transmission power by applying power control as expressed in relation (2). Assuming that all cells behave the same way, we update the interference $I_{c}$ and recompute $P_{e m_{j}}^{l}$ until we converge, after $S$ iterations, to a stable transmission power. Considering at the first iteration a noise limited network (i.e. $I_{c}=0$ ), users will converge quickly to a stable transmission power. The following algorithm explain these steps in detail.

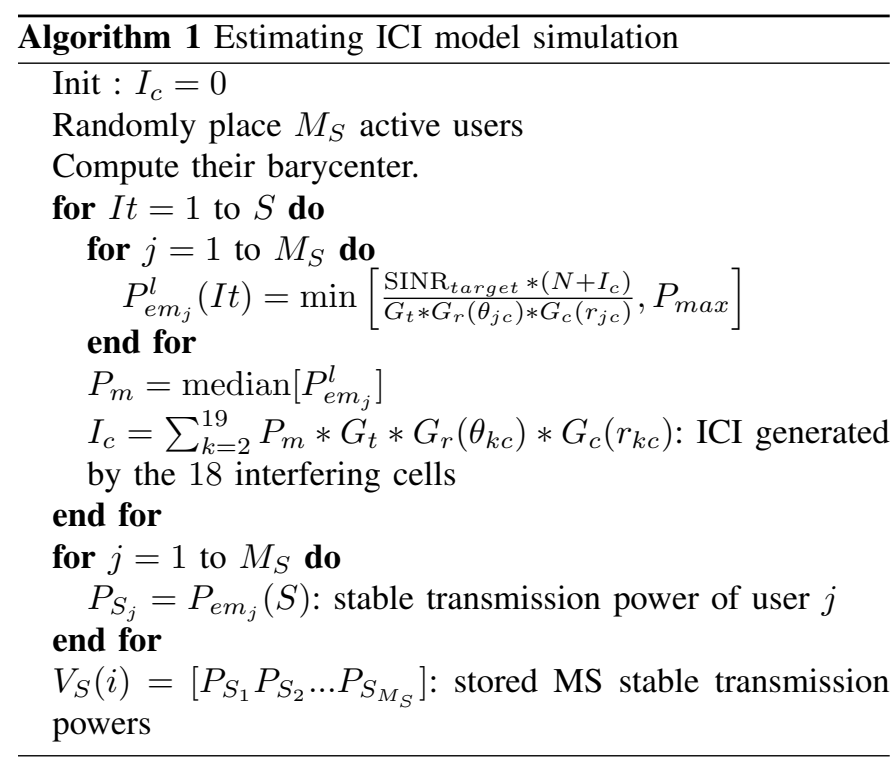

The Cumulative Distribution Function (CDF) of the stable transmission powers $P_{s_{j}}$ will allow us to evaluate the performance of the proposed method. In our case it concerns the distribution of MS stable transmission powers obtained (i) from our proposed model: $d_{M}$, and (ii) from Monte Carlo simulations: $d_{M C}$. For more noticeability, the comparison is done using two tests: i) the log ratio test, and ii) the KullbackLeibler divergence test.

- The log ratio test is a simple statistical ratio test, which compares two distributions using the logarithm of the ratio. In our case, the log ratio test $R$ is given by:

$$
R=\log \frac{\operatorname{Prob}\left(d_{M} \leq x\right)}{\operatorname{Prob}\left(d_{M C} \leq x\right)}
$$

if $R$ is less than 1 , or near to 0 (ideally) the distributions are similar.

- The Kullback-Leibler divergence test, mostly used in information theory, is a non-symmetrical measure of the difference between two probability distributions. It can be used to measure the consistency between $d_{M}$ and $d_{M C}$. The result, denoted $K L$, can be expressed as a function of the log ratio test as follows:

$$
K L=\operatorname{Prob}\left(d_{M} \leq x\right) * R
$$

The Kullback-Leibler test, as it is weighted by the CDF, is more representative of the distributions similarities, especially when the distributions are not uniform.

\begin{tabular}{|l|l|}
\hline cellular layout & Hexagonal grid,19 tri-sector cells. \\
\hline inter-site distance & $1732 \mathrm{~m}$ \\
\hline carrier frequency & $2.6 \mathrm{GHz}$ \\
\hline system bandwidth & $B=5 \mathrm{MHz}$ per sector \\
\hline $\begin{array}{l}\text { number of data } \\
\text { subcarriers }\end{array}$ & 300 \\
\hline $\begin{array}{l}\text { Total number of RB per } \\
\text { sector }\end{array}$ & 25 \\
\hline number of RB per user & 1 \\
\hline $\begin{array}{l}\text { number of active users } \\
M_{S}\end{array}$ & 25 \\
\hline TTI & $1 \mathrm{~ms}$ \\
\hline radio channel model & Okumura Hata for urban areas distance path \\
& loss: \\
& $G_{c}\left(r_{j c}\right)=10^{-a} / 10 * r_{j c}^{-b / 10}$. \\
\hline Antenna configuration & $a^{2}=136.7$ and $b=34.4$. \\
\hline BS antenna pattern & $G_{r}\left(\theta_{j c}\right)=-m i n\left[12 *\left(\frac{\theta_{j c}}{\theta_{3 d B}}\right)^{2}, A_{m}\right]$. \\
\hline User antenna gain & $\theta_{3 d B}=70^{\circ}, A_{m}=20 \mathrm{~dB}$ \\
\hline User power class & $G_{t}=0 \mathrm{dBi}$ \\
\hline $\begin{array}{l}\text { minumum distance } \\
\text { between user and BS }\end{array}$ & $30 \mathrm{~m}$ \\
\hline Thermal noise & $-174 \mathrm{dBm} / \mathrm{Hz}$ \\
\hline
\end{tabular}

TABLE I

SIMULATION PARAMETERS

\section{Performances of the Model}

In this section, the proposed method for ICI estimation is evaluated. We perform link-level simulations on the LTE SCFDMA uplink radio interface.

\section{A. Simulation parameters}

The parameters for uplink SC-FDMA system are mainly taken from [13]. Table I summarizes the most relevant parameters considered for our simulations.

Setting $B$ to $5 \mathrm{MHz}$, we obtain 300 subcarriers for data according to LTE standards [12]. Consequently, $25 \mathrm{RB}$ are available for uplink transmission every Time Transmission Interval (TTI) in each sector. The carrier frequency $f$ is equal to $2.6 \mathrm{GHz}$.

In the proposed method of ICI estimation, we have selected the median value for the virtual user transmission power instead of the mean value in order to achieve more accurate results. Indeed, the mean is a central tendency in statistics, which is reliable only in the presence of a symmetrical distribution, whereas the median is till reliable in presence of an asymmetrical distribution, since it is considered as a weighted arithmetic average [16]-[18].

In our case, the distribution of the MS transmission powers follows an asymmetrical distribution. Figure 3 represents the histogram of the users stable transmission powers after Monte Carlo simulations convergence. The asymmetry is caused by extreme values that correspond to some extreme users positions.

The method's performances are assessed by comparing the obtained stable transmission powers with those issued from Monte Carlo simulations, where the interference of each active 


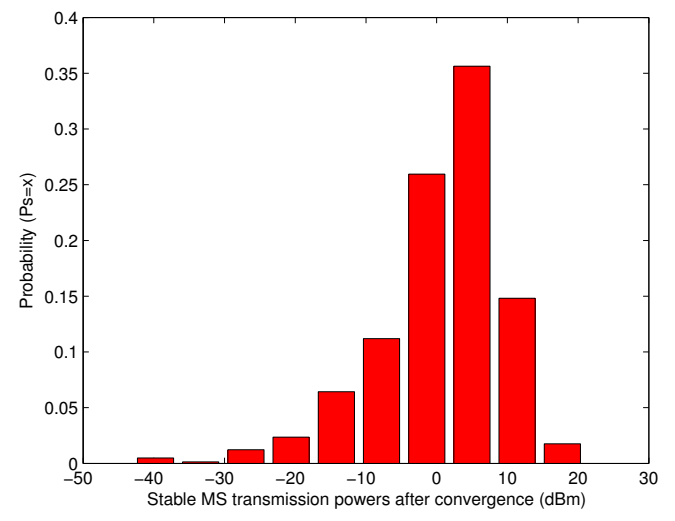

Fig. 3. Histogram of MS transmission powers after convergence

user is computed with $M_{T}$ random draws of interferers in each interfering sector.

To validate our model, we use the two tests defined before, and study the method in different and more realistic environments:

- PL: Path Loss using only the Okumura Hata model

- PL+Fad : Path Loss with Rayleigh fading of $\sigma_{\text {fading }}=1$

- PL+Fad+Shad : Path Loss with Rayleigh fading of

$\sigma_{\text {fading }}=1$, and Shadowing effect of standard deviation

$\sigma_{\text {shadowing }}=4 \mathrm{~dB}$

Many draws of Algorithm I and Monte Carlo simulations have been considered to obtain more representative results. The number of draws is denoted $M$ and set to $4.10^{5}$. Thanks to adequate initialization of $I_{c}$, the stable transmission powers are obtained after $S$ less than 5 iterations.

\section{B. Robustness of the model}

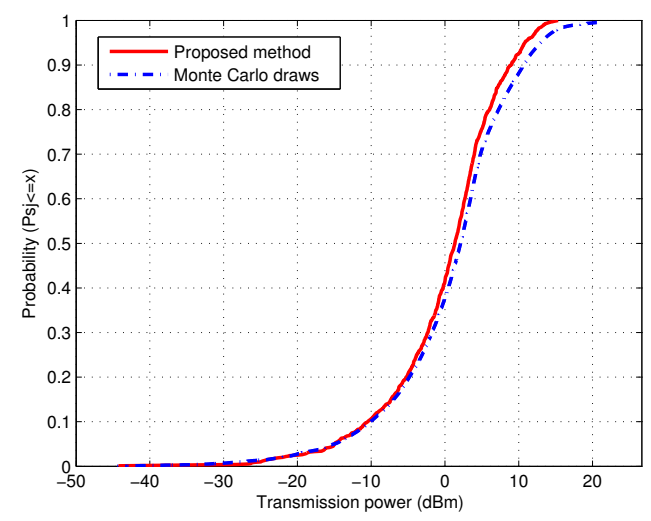

Fig. 4. CDF of MS transmission powers

Figure 4 represents the two CDF obtained, after convergence of MS transmission powers, by our proposed method and by Monte Carlo simulations. For both methods Okumura Hata propagation model without fading and shadowing was firstly used.

\begin{tabular}{|l|l|l|l|}
\hline \multirow{2}{*}{ Power $(\mathrm{dBm})$} & \multicolumn{3}{|c|}{$\mathrm{R}$} \\
\cline { 2 - 4 } & \multicolumn{3}{|c|}{$\sigma_{\text {fading }}=1, \sigma_{\text {shadowing }}=4 \mathrm{~dB}$} \\
\hline$[[\leq-30$ & 0.14 & 0.11 & 0.122 \\
\hline$[-30 ;-25]$ & 0.048 & 0.063 & 0.11 \\
\hline$[-25 ;-20]$ & 0.04 & 0.009 & 0.099 \\
\hline$[-20 ;-15]$ & 0.024 & 0.026 & 0.09 \\
\hline$[-15 ;-10]$ & 0.022 & 0.033 & 0.078 \\
\hline$[-10 ;-5]$ & 0.02 & 0.024 & 0.068 \\
\hline$[-5 ; 0]$ & 0.03 & 0.042 & 0.06 \\
\hline$[0 ; 5]$ & 0.043 & 0.042 & 0.049 \\
\hline$[5 ; 10]$ & 0.026 & 0.031 & 0.037 \\
\hline$[10 ; 15]$ & 0.014 & 0.018 & 0.028 \\
\hline$[15 ; 21]$ & 0.003 & 0.0042 & 0.015 \\
\hline
\end{tabular}

TABLE II

$R$ OBTAINED BY LOG RATIO TEST

1) Complexity gain: The two curves are obtained with $M \times M_{S}$ and $M \times M_{T} \times M_{S}$ points respectively. Actually, for each Monte Carlo simulation, $M_{T}$ iterations are run additionally to drop interferer users in each interfering sector. This last parameter $M_{T}$ represents the computation complexity gain of our proposed method as it does not need to drop interfering users to evaluate intercell interference. Setting $S I N R_{\text {target }}=$ $3 \mathrm{~dB}$, less than $2 \%$ of users are in outage, whereas user transmission powers values vary between $-48 \mathrm{dBm}$ and $21 \mathrm{dBm}$, which respect the standard transmission power interval given in [19]. The corresponding throughput per user is $360 \mathrm{kbps}$.

2) Introduction of fast fading and shadowing: Using the $\log$ ratio test in different environments, table II summarizes the most representative results identified by significant probabilities, i.e. MS transmission powers $\geq-30 \mathrm{dBm}$, obtained . The gap between the two CDF for lower MS transmission powers is larger than the observed one for high transmission powers, which becomes close to zero. Moreover, the complex environment increases this gap. However, the largest value observed is 0.14 . Therefore, as it is very lower than 1 , the two CDF can be considered as similar and our model is valid.

The Kullback-Leibler divergence test, as it takes into account the MS transmission power CDF, softens the differences between the results issued from both methods (see Table III) in the lowest transmission powers area. It enhances that in the major part of the time, when MS transmission powers are higher than $-15 \mathrm{dBm}$, the two MS stable transmission powers CDF's are similar.

3) Variation of the fast fading and shadowing environment parameters: Using the Kullback-Leibler divergence test, we take different values for shadowing and fast fading parameters. The variation of these parameters, if it keeps the distributions similarity, will confirm the model's validity. Figure 5 summarizes the simulations results curves. As they come from the product of a decreasing function $(R)$ and an increasing function $\left(\operatorname{Prob}\left(d_{M} \leq x\right)\right)$, all curves have the same behavior: first they increase, reach a maximum value (that remains so small that it respects the log ratio validity test) and then decrease.

The maximum Kullback-Leibler test in the path loss case 


\begin{tabular}{|l|l|l|l|}
\hline \multirow{2}{*}{ Power $(\mathrm{dBm})$} & \multicolumn{3}{|c|}{ KL } \\
& \multicolumn{3}{|c|}{$\sigma_{\text {fading }}=1, \sigma_{\text {shadowing }}=4 \mathrm{~dB}$} \\
\cline { 2 - 4 } & PL & PL + Fad & PL+Fad+shad \\
\hline$\leq-30$ & 0.0016 & 0.0002 & 0.024 \\
\hline$[-30 ;-25]$ & 0.0028 & 0.0002 & 0.04 \\
\hline$[-25 ;-20]$ & 0.0012 & 0.00063 & 0.057 \\
\hline$[-20 ;-15]$ & 0.0027 & 0.0009 & 0.064 \\
\hline$[-15 ;-10]$ & 0.0019 & 0.037 & 0.063 \\
\hline$[-10 ;-5]$ & 0.0037 & 0.049 & 0.057 \\
\hline$[-5 ; 0]$ & 0.0086 & 0.014 & 0.052 \\
\hline$[0 ; 5]$ & 0.012 & 0.022 & 0.044 \\
\hline$[5 ; 10]$ & 0.024 & 0.025 & 0.035 \\
\hline$[10 ; 15]$ & 0.011 & 0.022 & 0.027 \\
\hline$[15 ; 21]$ & 0.005 & 0.006 & 0.014 \\
\hline
\end{tabular}

TABLE III

$K L$ OBTAINED By KULLBACK-LEIBLER TEST

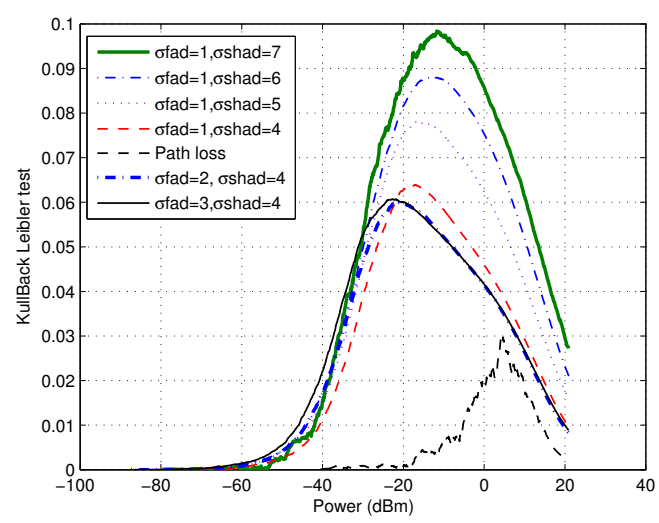

Fig. 5. Kullback-Leilbler test curves

was less than 0.03 . When the fast fading and the shadowing effects are considered, we observe some fluctuations. The maximum Kullbach-Leibler divergence test in presence of fast fading of $\sigma_{\text {fading }}=1$ and shadowing of $\sigma_{\text {shadowing }}=4 \mathrm{~dB}$ is less than 0.07. Moreover, varying fast fading standard deviation $\sigma_{\text {fading }}$ from 1 to 3 does not influence much. The curves remain nearly identical. On the other hand, varying the shadowing parameter changes the simulation results. Setting $\sigma_{\text {fading }}$ at 1 and varying $\sigma_{\text {shadowing }}$ from 4 to $7 \mathrm{~dB}$ makes the maximum Kullbach-Leibler distance increase from 0.06 to 0.1 . Nevertheless, the model's robustness is confirmed as the Kullbach-Leibler divergence test results remains very small and respects the log ratio validity tests. This confirms the validity of the proposed model in different environments and its robustness against the environment parameters variations.

\section{CONClusion}

Power control applied on the uplink to the mobile terminals transmission power, based on a SINR threshold, reduces efficiently the inter cell interference. This reduction comes at the expense of a higher complexity in the derivation of ICI. The method we proposed to estimate the inter cell interference is less heavy and greedy in computation than the classical Monte Carlo method. Actually, it does not drop users in the interfering sectors as Monte Carlo method requires.
We have shown that a single virtual point, situated at the barycenter of each the concerned interfering sector, allows us to precisely evaluate the ICI. This virtual interferer, transmits at the median power of the sector's active users assuming that all neighboring cells behave the same way than the central cell. This equivalent median power creates, in any environment, an intercell interference at the central base station identical to that derived with random interfering users obtained from Monte Carlo simulations. This method reduces computation of the ICI, and complexity of planning and evaluation simulators. Future work will consist in developing a closed formula for ICI estimation, in order to validate analytically the model proposed in this paper.

\section{REFERENCES}

[1] 3GPP TR 25.913, "Requirements for evolved utra (E-UTRA) and evolved utran (E-UTRAN) (release 9)", 3GPP TSG RAN, version 9.00, 2009.

[2] J. C. Ikuno, M. Wrulich, and M. Rupp, , "System level simulation of LTE networks", in Proc.IEEE Vehicular Technology Conference, ( Taipei, Taiwan), May, 2010.

[3] J. Heyman, "Intercell interference management in an OFDM-based downlink", Ph.D. dissertation, Institutionen fr systemteknik Department of Electrical Engineering, 2006.

[4] J. Lafuente-Martinez, A. Hernandez-Solana, I. Guio, A. Valdovinos, "Radio resource strategies for uplink inter-cell interference fluctuation reduction in SC-FDMA cellular systems", Wireless Communications and Networking Conference, (Cancun, Quintana Roo), Mar, 2011.

[5] L. Cao,L. Zhong,H. Lei,Y. Wang,Y. Chang,D. Yang,"Uplink Power Control for an SC-FDMA Mobile Cellular System", Vehicular Technology Conference, 2008.

[6] F.D. Calabrese, M. Anas, C. Rosa, P.E. Mogensen, K.L. Perersen, "Performance of a Radio Resource Allocation Algorithm for UTRAN LTE Uplink", Vehicular Technology Conference, p, 2895-2899, Apr, 2007.

[7] M. Pischella and J.-C. Belfiore, "Optimal power allocation for downlink cooperative cellular networks", Proc. of Vehicular Technology Conference, (Dublin, Ireland), Apr, 2007.

[8] S. Coleri, M. Ergen, A. Puri, A. Bahai, "Channel Estimation Techniques Based on Pilot Arrangement in OFDM Systems", IEEE transaction on broadcasting, vol 48, Sep, 2002.

[9] Y. S. Yameogo, J. Palicot, L. Cariou, "Mobile radio channels' Estimation for SC-FDMA systems by means of adequate Noise and Inter-carrier Interference filtering in a transformed domai”, IEEE Global Communications Conference, (Miami, USA),Dec, 2010.

[10] I. Viering, A. Klein,M. Ivrlac, M. Castaneda, "On Uplink Intercell Interference in a Cellular System", IEEE International Conference, Jun, 2006.

[11] S.E. Elayoubi, O. Ben Haddada, "Uplink Intercell Interference and Capacity in 3G LTE systems", IEEE International Conference On Networks, Nov. 2007.

[12] 3GPP TR 36.211, "E-UTRA ; physical channels and modulation (release 8)", 3GPP TSG RAN, version V8.6.0, 2008.

[13] 3GPP TR 36.942, v9.2.0, "E-UTRA; Radio Frequency (RF) system scenarios (Release 9)", Dec 2010.

[14] X. Lu, E. Kunnari, J. Leinonen, O. Piirainen, M. Vainikka, M. Juntti, "LTE uplink power control and base station antenna down tilt a 3d channel model)", wireless conference, Apr, 2010.

[15] Y. Okumura, E. Ohmori, T. Kawano, K. Fukuda, "Field Strength and Its Variability in VHF and UHF Land-Mobile Radio Service", Review of the Electrical communication Laboratory, pp. 825-873, Oct, 1968,

[16] A. Papoulis, S. U. Pillai, "Probability, Random Variables and Stochastic Processes", McGraw, 2002.

[17] C.A. Marazzi, "The truncated mean of an asymmetric distribution", Computational statistics and data analysis.

[18] S. Mishra, "Median as a weighted arithmetic mean of all sample observations", Economic Bulletin.

[19] 3GPP TR 36.101, "E-UTRA ; User Equipement (UE) radio transmission and reception (release 10)", 3GPP TSG RAN, version V10.3.0,Jun 2011. 